PROTEOSTASIS

\section{Relieving stress}

Mol. Cell https://doi.org/10.1016/j.

molcel.2019.09.026 (2019)

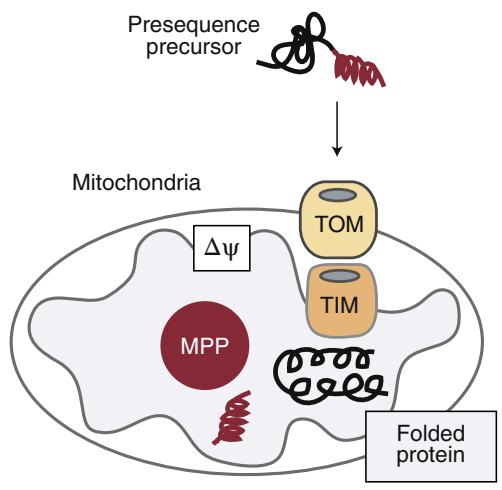

Credit: Cell Press

Nuclear-encoded mitochondrial proteins are directed to their destination by $\mathrm{N}$-terminal leader peptides, which are removed by the presequence protease MPP, localized in the mitochondrial matrix. Improper processing of precursor proteins through loss of MPP function is thought to impair protein stability. Using a yeast strain containing a temperature-sensitive MPP, PovedaHuertes et al. revealed that deactivation of MPP at higher temperatures induced abnormal accumulation of unprocessed precursor proteins that formed insoluble aggregates inside mitochondria but did not alter mitochondrial function or cell survival. RNA-seq analysis revealed that genes associated with protein folding and refolding were upregulated. The authors used a synthetic lethality assay to identify nuclear protein Rox1 as a critical mediator of survival upon loss of MPP activity. Loss of MPP function resulted in the translocation of Rox1 into the mitochondria, where it binds mitochondrial DNA, thereby stabilizing de novo synthesized DNA and enhancing transcription and translation. This study reveals a new early mitochondrial unfolding response and stresses the role of intracellular communication in regulating proteostasis. YS

https://doi.org/10.1038/s41589-019-0419-y

\section{ENZYMOLOGY}

\section{lodine goes viral}

Nat. Chem. https://doi.org/10.1038/s41557-0190349-z (2019)

Flavin-dependent halogenases (FDHs), which catalyze the formation of carbonhalogen bonds, are most often of bacterial or fungal origin and are typically selective for either chlorine or bromine. Seeking new FDHs, Gkotsi et al. performed a sequence alignment of all characterized FDHs, from which they identified a previously unappreciated conserved sequence motif. Genome mining with this motif as a probe led them to focus on the halogenase VirX1 from a cyanobacteria-infecting virus. In vitro, VirX1 exhibited an unusual preference for iodide donors over chloride or bromide, along with promiscuous activity for numerous organic acceptor substrates. The structure of VirX1 is overall similar to those of other FDHs, but has an especially wide opening to an already expanded substrate-binding cavity, enabling it to accommodate larger halides and a broad scope of substrates. Although certain other halogenases exhibit some level of iodination
CELL DEATH

\section{A back-up plan}

Ferroptosis is a form of iron-dependent cell death caused by elevated lipid peroxidation. Glutathione peroxidase 4 (GPX4)-mediated conversion of phospholipid hydroperoxides into non-toxic lipid alcohols is thought to mitigate ferroptosis, and GPX4 inhibitors promote ferroptosis in certain cancer cells. To explore alternative mechanisms to prevent ferroptosis, Besuker et al. performed a synthetic lethal CRISPR-Cas screen in a ferroptosis-resistant cell line, and Doll et al. screened for genes that could complement GPX4 inhibition. In both cases, they revealed that an NADH-dependent coenzyme $\mathrm{Q}$ oxidoreductase, ferroptosis-suppressor protein 1 (FSP1), protected cells against ferroptosis. FSP1 required N-myristoylation and plasma-membrane association for its antiferroptotic activity, and it reduced coenzyme Q10 in the lipid bilayer to (re)generate radical-trapping antioxidants that inhibit lipid peroxidation. The increased expression of FSP1 was correlated with ferroptosis resistance in numerous cancer cell lines, and inhibition of FSP1 resulted in increased sensitivity to ferroptosis inducers. Altogether, these findings reveal a parallel pathway that mitigates ferroptosis together with GPX4. ability, VirX1 represents the first example of a halogenase with such a preference. This specialized activity of VirX1, coupled with its broad substrate scope, provides a new potential biocatalytic route to iodinecontaining compounds.

https://doi.org/10.1038/s41589-019-0416-1

\section{GPCRS}

\section{Choosing a mating site}

PLOS Biol. https://doi.org/10.1371/journal. pbio.3000484 (2019)

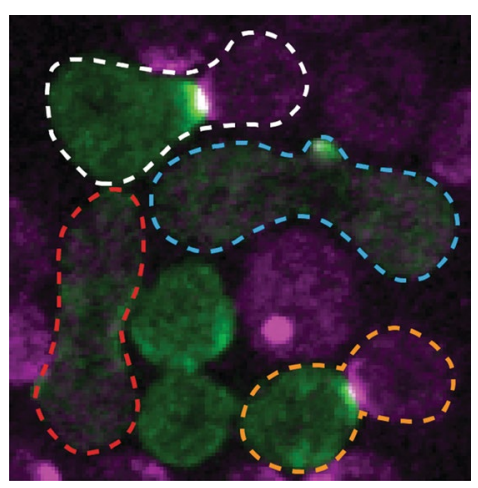

Credit: PLoS

Sensing of chemical gradients is important for cells to orient their growth and migration. In the yeast Saccharomyces cerevisiae, cells of opposite mating types secrete pheromones that act on their partners through a G-protein-coupled receptor (GPCR) in both cell types. GPCR activation leads to transcription of mating-related genes and concentration of the small $\mathrm{G}$ protein $\mathrm{Cdc} 42$, a regulator of cell polarity, to a distinct site on the cell cortex to orient the cytoskeleton. To understand how yeast cells sense the pheromone gradient to direct polarization, Henderson et al. performed various imaging and modeling studies, revealing a spatial mechanism that senses the ratio of occupied versus unoccupied receptors, amplifying the pheromone gradient. An exploratory mechanism then samples ligand concentrations across the cell during an indecisive phase, in which the polarity site wanders around the cortex, and is followed by a committed phase, in which cells are prepared for mating. These mechanisms act to align the Cdc42 polarity site and improve the search for a mating partner even in situations of non-uniform GPCR distribution.

https://doi.org/10.1038/s41589-019-0418-z

Mirella Bucci, Caitlin Deane, Grant Miura and Yiyun Song 